\title{
B-parameters of 4-fermion operators from lattice QCD
}

\author{
Rajan Gupta ${ }^{\text {a* }}$

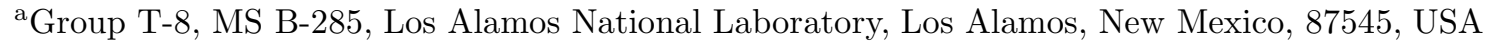

This talk summarizes the status of the calculations of $B_{K}, B_{7}, B_{8}$, and $B_{s}$, done in collaboration with T. Bhattacharya, G. Kilcup, and S. Sharpe. Results for staggered, Wilson, and Clover fermions are presented.

\section{INTRODUCTION}

Reliable estimates of the matrix elements of 4 -fermion operators between hadronic states are essential in order to quantify strong interaction corrections to weak processes. Here we report on calculations of mixing elements of (i) $\Delta s=2$ operators between $K^{0}$ and $\bar{K}^{0}$ states $\left(B_{K}\right)$ that arise in the calculation of the $\mathrm{CP}$ violation parameter $\epsilon$. The value of $B_{K}$ is an essential input in pinning down the Wolfenstein parameters $\rho$ and $\eta$ in the CKM matrix. (ii) The strong and electromagnetic penguin operators needed to predict $\epsilon^{\prime} / \epsilon\left(B_{6}\right.$ and $\left.B_{8}\right)$. In particular, we consider $B_{8}$, which is phenomenologically important since a smaller value means a larger $\epsilon^{\prime} / \epsilon$. (iii) The $S+P$ operators needed in the study of the lifetime differences of $\mathrm{B}$ mesons $\left(B_{s}\right)$.

\section{STAGGERED RESULTS}

Staggered fermions are the method of choice for calculating kaon matrix elements as they respect the continuum chiral Ward identities. Our results for $B_{K}, B_{7}$, and $B_{8}$ have recently been given in 11. They are based on the same numerical data first presented at LATTICE 93 by Sharpe [2]. As explained in [1], even though the statistical quality of the data is meager by present standards, the largest source of error in the quenched staggered theory is a systematic one - the dependence of $B_{K}$ on the lattice operator. The second new feature of the analysis, compared to [2], is a better understanding of the matching between lattice and continuum operators using tadpole improved 1-loop perturbation theory, i.e. the horizontal matching

\footnotetext{
*Work supported by DoE Grand Challenges award.
}

explained in [3].

The data for both "smeared" $(\beta=6.0,6.2)$ and "unsmeared" $(\beta=6.0,6.2,6.4)$ operators are shown in Fig. 1. (See [1] for definition of these operators.) The extrapolation to $a=0$ is done assuming that only the leading correction $O\left(a^{2}\right)$ contributes (the absence of the linear $O(a)$ term is expected theoretically [2], and has been confirmed numerically 顿). The results are (for $\left.q^{*}=1 / a\right)$

$$
\begin{aligned}
& B_{K}(N D R, 2 \mathrm{GeV})=0.63(2) \text { unsmeared } \\
& B_{K}(N D R, 2 \mathrm{GeV})=0.60(2) \text { smeared }
\end{aligned}
$$

For the final value we take the mean

$B_{K}(N D R, 2 \mathrm{GeV})=0.62 \pm 0.02 \pm 0.02$

where the second error covers the spread due to the operator dependence.

The difference between the central values for smeared and unsmeared operators is $\sim 0.025$. While our data is certainly not good enough to argue that this difference is significant, a difference of similar size has been reported by JLQCD [4]. This difference is an artifact of keeping only an $a^{2}$ correction term in the $a=0$ extrapolation as shown by the following argument. Consider two discretizations, $\mathcal{O}_{1}$ and $\mathcal{O}_{2}$, of any 4 -fermion operator. Let the typical lattice momenta $q^{*}$ associated with their lattice measurement be $K_{1} / a$ and $K_{2} / a$ respectively. Then, using the "horizontal" matching to the continuum scheme defined in [3], the results at $\mu=K_{1} / a$ are related as

$$
\begin{aligned}
\mathcal{O}_{1}(\mu) & =\mathcal{O}_{2}(\mu)\left(\alpha\left(K_{2} / a\right) / \alpha\left(K_{1} / a\right)\right)^{-\gamma_{0} / 2 \beta_{0}} \\
& +\left\{1+X \alpha^{2}(q *)+Y a^{2}+\ldots\right\} .
\end{aligned}
$$

Since the factor $\alpha\left(K_{2} / a\right) / \alpha\left(K_{1} / a\right) \rightarrow 1$ as $a \rightarrow 1$, the two operators should give the same result in 


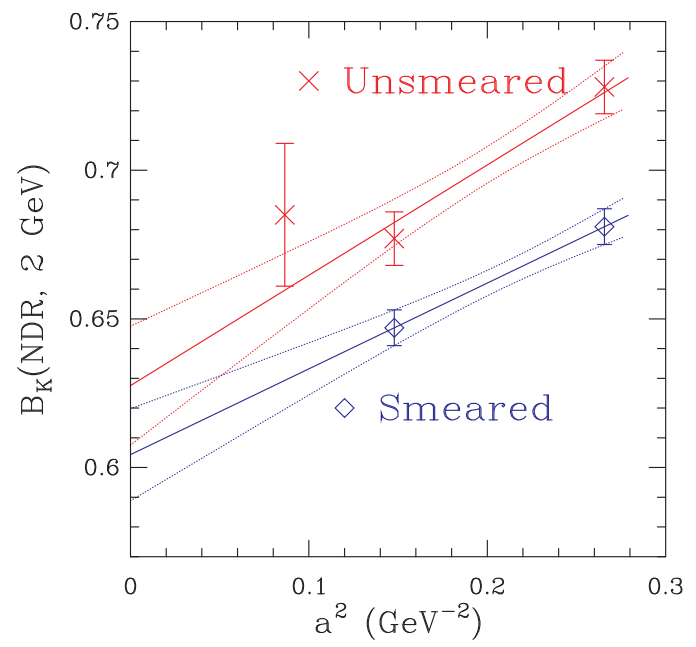

Figure 1. The data for $B_{K}(\mathrm{NDR}, 2 \mathrm{GeV})$ as a function of lattice spacing $a^{2}$, along with a linear extrapolation to $a=0$, for the smeared and unsmeared operators.

the continuum limit provided the extrapolation is done including factors of both $O(a)$ and $O(\alpha)$.

To convert the quenched result in Eq. 2 to the renormalization group invariant quantity $\widehat{B}$, one can proceed in two ways: use the $n_{f}=0$ or the $n_{f}=4$ values for $\alpha_{s}, \beta_{0}, \beta_{1}, \gamma_{1}$. The quenched result, using $\alpha(2 \mathrm{GeV})=0.19$, is

$\widehat{B}_{K}=0.86 \pm 0.03 \pm 0.03$

Interestingly, using $n_{f}=4$ values for $\beta_{0}, \beta_{1}, \gamma_{1}$ and $\alpha \frac{(4)}{M S}(2 \mathrm{GeV})=0.3$ also gives the same value. However, there is an uncertainty of $\sim 0.05$ in such a conversion. What we really predict is the quenched result Eq. 2.

\subsection{Electromagnetic penguins}

The quantity that enters in the standard model calculation of $\epsilon^{\prime} / \epsilon$ is $B_{8}$ evaluated at say $\mu=2$ $\mathrm{GeV}$ [5]. To get this from lattice simulations one needs to calculate the matrix elements of both $O_{7}$ and $O_{8}$ as these mix under a scale evolution.

Our current calculations use 1-loop matching factors for the 4-fermion operators. As explained in 価, if the discretization of operators is such that $Z_{P}$ for the pseudoscalar bilinear is large, then the mixing contribution due to the $P \otimes P$ term can be even larger than the tree level result. In such a case the 1-loop determination of the matching $Z$ 's is inadequate, and no results for $B_{7}$ or $B_{8}$ can be extracted. This is true for the unsmeared Landau gauge operators that we have used and probably also for the gauge invariant operators used by JLQCD 泪 as they have the same $Z_{P}$. On the other hand, the 1-loop perturbative value for $Z_{P}$ for the smeared operators is much smaller, and consequently results are independent of $q^{*}$ to within $10 \%$ as shown in Table 11. (The failure of 1-loop $Z$ 's for the unsmeared operators shows up as a large dependence of $B$-parameters on $q^{*}$; for example the results even change sign between $q^{*}=\pi / a$ and $1 / a$ [1].)

The shaky part of this analysis is that the data at two values of $a$ are extrapolated using just the lowest order $\left(a^{2}\right)$ correction. Since these correction are large, further checks of these first results are needed.

\section{WILSON FERMIONS}

Our results with Wilson fermions are exploratory. They have been obtained at just $\beta=$ 6.0 , albeit on large lattices and with high statistics [3]. The goal has been to understand systematic errors, in particular the question of bad chiral behavior of matrix elements induced by the mixing with wrong chirality operators.

The general form of the kaon matrix elements, as predicted by $\chi \mathrm{PT}$, is

$$
\begin{aligned}
& \frac{\left\langle\overline{K^{0}}\left(p_{f}\right)|\mathcal{O}| K^{0}\left(p_{i}\right)\right\rangle}{(8 / 3) f_{K, \text { phys }}^{2}}=\alpha+\beta m_{K}^{2}+\gamma p_{i} \cdot p_{f}+ \\
& \delta_{1} m_{K}^{4}+\delta_{2} m_{K}^{2} p_{i} \cdot p_{f}+\delta_{3}\left(p_{i} \cdot p_{f}\right)^{2}+\ldots
\end{aligned}
$$

where we ignore chiral logarithms and terms proportional to $\left(m_{s}-m_{d}\right)^{2}$. The former are difficult to distinguish numerically from the terms we include, while the latter we expect to be small, especially for the range of quark masses studied.

For $B_{K}$, chiral symmetry predicts that $\alpha, \beta$, and $\delta_{1}$ are zero. With Wilson fermions, the mixing with wrong chirality operators generates these terms and, in addition, the allowed terms $\gamma, \delta_{2}, \delta_{3}$ get contributions that have to be eliminated. 


\begin{tabular}{lrrrr}
\hline Operator & $q^{*}$ & $\beta=6.0$ & $\beta=6.2$ & $a=0$ \\
\hline$B_{7}^{3 / 2}$ & $1 / a$ & $0.989(05)$ & $0.823(16)$ & $0.62(3)$ \\
$B_{7}^{3 / 2}$ & $\pi / a$ & $1.085(06)$ & $0.903(14)$ & $0.67(3)$ \\
$B_{8}^{3 / 2}$ & $1 / a$ & $1.240(06)$ & $1.030(16)$ & $0.77(4)$ \\
$B_{8}^{3 / 2}$ & $\pi / a$ & $1.288(06)$ & $1.076(17)$ & $0.81(4)$ \\
\hline
\end{tabular}

Table 1 for $B_{7}^{3 / 2}(\mathrm{NDR}, 2 \mathrm{GeV})$ and $B_{8}^{3 / 2}(\mathrm{NDR}, 2 \mathrm{GeV})$, at the physical kaon mass, using smeared operators. The last column gives the result of linear extrapolation in $a^{2}$.

With 1-loop improved operators these artifacts are $O\left(\alpha_{s}^{2}\right)$, but nevertheless overwhelm the signal. Our approach is to first remove $\alpha, \beta, \delta_{1}$ by studying the momentum dependence, and secondly, roughly estimate the artifacts in $\gamma, \delta_{2}, \delta_{3}$ using the fact that $\alpha, \beta, \delta_{1}$ have to be zero. Putting all these together gives, without extrapolation in $a,[3]$

$B_{K}(N D R, 2 \mathrm{GeV})=0.74 \pm 0.04 \pm 0.05$

where the second error is an estimate of the residual contamination due to bad chiral behavior.

Results for the electromagnetic penguins operators, in the NDR scheme at $\mu=2 \mathrm{GeV}$, are [3]

$B_{7}^{3 / 2}=0.58 \pm 0.02$ (stat) ${ }_{-0.03}^{+0.07}$ (pert),

$B_{8}^{3 / 2}=0.81 \pm 0.03$ (stat) ${ }_{-0.02}^{+0.03}$ (pert).

The "perturbative error" reflects the dependence of the results on the choice of $\alpha_{s}$ used in the matching of continuum and lattice operators, and is comparable to or larger than the statistical errors. A recent calculation of these by the APE collaboration using non-perturbative matching coefficients and the $C_{S W}=1$ clover action suggests that the errors in the 1-loop mixing coefficients may be far more severe [6].

The final quantities we consider are $B_{S} \equiv B_{4}^{+}$ and the related parameter $B_{5}^{+}$as defined in [3]. The matrix elements we require are for $\bar{b} s$ mesons. The best we can do with present data is to give the result for $m_{b} \sim m_{c}$,

$B_{4}^{+}(\mathrm{NDR}, 1 / a)=0.80 \pm 0.01$ (stat),

$B_{5}^{+}(\mathrm{NDR}, 1 / a)=0.94 \pm 0.01$ (stat) .

A second limitation is that these results are at $\mu=1 / a=2.33 \mathrm{GeV}$, the scale at $\beta=6.0$, be- cause the two-loop anomalous dimension matrix needed to run to $2 \mathrm{GeV}$ has not been calculated.

\section{CLOVER FERMIONS}

The analysis of data with clover fermions is preliminary. The same 170 lattices used in the study with Wilson fermions [3] are analyzed with tree-level tadpole improved clover action $\left(C_{S W}=\right.$ 1.4785). At this point we have a few qualitative statements about the data.

The statistical fluctuations in the matrix elements of $\mathcal{S}, \mathcal{P}, \mathcal{A}, \mathcal{V}, \mathcal{T}$ operators are much larger compared to those with Wilson fermions. Curiously, these fluctuations cancel in the five operators $O_{1}^{+} \ldots O_{5}^{+}$discussed above.

The 1-loop mixing factors for $O_{7}^{3 / 2}$ and $O_{8}^{3 / 2}$ are too large and the calculation fails. The reason is the increase in $Z_{P}$ with $C_{S W}$.

The dominant artifacts in $B_{K}, \alpha$ and $\beta$, are roughly a factor of five smaller compared to Wilson fermions. As a result, the lattice value of $B_{K}$ using the 1-loop improved operator improves from -0.30 with Wilson fermions to 0.50 with clover. This suggests that a large part of chiral violations is an $O(a)$ effect.

\section{REFERENCES}

1. Kilcup, Gupta, Sharpe, hep-lat/9707006.

2. S. Sharpe, Nucl. Phys. (Proc. Supp.) B34 (1994) 403.

3. R. Gupta, T. Bhattacharya, S. Sharpe, Phys. Rev. D55 (1997) 4036.

4. JLQCD Collaboration, Nucl. Phys. (Proc. Supp.) B53 (1997) 341; S. Aoki, ibid.

5. A. Buras and R. Fleischer, hep-ph/9704376.

6. A. Vladikas, Talk at LATTICE 97, ibid. 